\section{Caracterización de pacientes hospitalizados con neoplasia activa y diagnóstico de enfermedad tromboembólica venosa mediante la puntuación de Khorana en el Instituto Nacional del Cáncer}

\author{
VERÓNICA KRAMER ${ }^{1, a}$, MACARENA KLEIN ${ }^{3, d}$, \\ FERNANDO CEVALLOS ${ }^{3, \mathrm{~d}}$, MARCELA CÁRCAMO ${ }^{2, \mathrm{~b}, \mathrm{c}}$
}

\section{Khorama score as a predictive tool for thromboembolic events in high risk patients}

Background: There is an established relation between cancer and the development of thromboembolic venous disease. Khorana et al developed a predictive score using clinical characteristics and laboratory values to stratify patients according to their risk. Aim: To characterize using Khorana score, patients with active cancer and a diagnosis of thromboembolic disease during hospitalization in an oncology hospital. Material and Methods: Review of records of the pharmacy of the hospital, selecting patients who received heparin in therapeutic doses during their hospitalization. Using laboratory values available in the medical records, the Khorama score was calculated. Results: Twenty seven patients with thromboembolic events, aged $60 \pm 2$ years (56\% males) were selected for the study. Eighty percent of them were catalogued in the intermediate and high risk group according to Khorana score. Conclusions: Khorana score is a simple scale that might be useful to establish early prophylactic measures in patients with high risk for thromboembolic events, especially those with cancer.

(Rev Med Chile 2016; 144: 593-597)

Key words: Neoplasms; Risk Factors; Thrombosis: Thromboembolism; Venous Thrombosis.

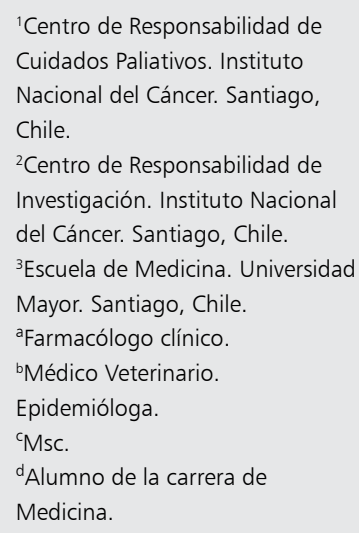

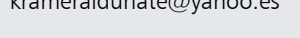

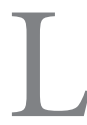

a enfermedad tromboembólica (ETE), patología que comprende tanto la trombosis venosa profunda como el tromboembolismo pulmonar, es una condición grave que puede llegar a complicar la evolución de los pacientes hospitalizados ${ }^{1}$. Se estima que esta condición puede afectar entre $5 \%$ y $15 \%{ }^{2}$ de este tipo de pacientes, contribuyendo hasta $5 \%$ de las muertes que ocurren en un hospital ${ }^{3}$.

La asociación entre el cáncer y la ETE está bien establecida: el riesgo se multiplica por 4 en los pacientes con cáncer. Hasta $20 \%$ de los pacientes con cáncer desarrollan ETE, que es reconocida como una de las principales causas de muerte en estos pacientes ${ }^{4-7}$. Además, existen factores de riesgo asociados para cáncer-ETE, como por ejemplo, el sitio de cáncer, presencia de enfermedad metastásica y el uso de terapia antineoplásica, incluida la quimioterapia, hormonas, cirugía, pacientes ambulatorios u hospitalizados entre otros, lo que a su vez determina las diferentes frecuencias reportadas de ETE, según la población estudiada ${ }^{8,9}$.

Los pacientes con cáncer en tratamiento activo tienen un riesgo mayor de desarrollar ETE, 
alcanzando 4,1 veces mayor riesgo de trombosis y en los pacientes tratados con quimioterapia, el riesgo aumenta a $6,5^{10}$.

La profilaxis para ETE puede reducir la trombosis venosa, embolismo pulmonar y embolismo pulmonar fatal en pacientes de alto riesgo, como lo son los hospitalizados o postoperados, especialmente la subpoblación de pacientes con cáncer ${ }^{11}$.

Es así como las nuevas guías de la Academia Americana de Oncología Clínica (ASCO) ${ }^{12}$ y $\mathrm{Na}$ tional Comprehensive Cancer Network (NCCN) ${ }^{13}$ recomiendan la tromboprofilaxis en pacientes con cáncer, tanto en pacientes hospitalizados, como ambulatorios, en ausencia de hemorragia u otras contraindicaciones.

Ante ello, se desarrolló un modelo de riesgo para predecir la tasa de tromboembolismo venoso, identificando cinco parámetros clínicos y de laboratorio que independientemente predicen el riesgo de ETE en pacientes con cáncer ${ }^{14}$.

Debido a lo anteriormente expuesto, es que este estudio quiso caracterizar, mediante la puntuación de Khorana, a pacientes con neoplasia activa y diagnóstico de ETE durante su hospitalización en el Instituto Nacional del Cáncer (INC), Santiago de Chile.

\section{Material y Método}

Diseño: Serie de casos.

Se accedió al sistema informático de Farmacia del Instituto Nacional del Cáncer (INC). De la base de datos del período de enero a diciembre de 2009, se seleccionaron a todos los pacientes hospitalizados en que se les indicó heparina en dosis terapéutica (pacientes con sospecha de ETE). Los criterios de inclusión fueron pacientes en los que se confirmó ETE basado en pruebas de imagen, y que estaban diagnosticados de un cáncer activo en el momento del evento, independiente del período de tratamiento o tipo de terapia recibida. Se excluyó a los pacientes con indicación de heparina por fibrilación auricular y pacientes que se descartó ETE.

Para la confección de la base de datos durante este período, se incluyeron las siguientes variables: rol único tributario (RUT), número de ficha, edad, sexo, diagnóstico oncológico, tipo de ETE, recuento de plaquetas, hemoglobina, leucocitos e índice de masa corporal (IMC).
Este estudio fue aprobado por el Centro de Responsabilidad de Investigación del Instituto Nacional del Cáncer.

Para la aplicación del modelo de Khorana, se realizó una combinación de 5 variables: sitio primario del cáncer, nivel de plaquetas pre quimioterapia de $350 \times 10^{9} / \mathrm{L}$ o más, niveles de hemoglobina menores que $100 \mathrm{~g} / \mathrm{L}(10 \mathrm{~g} / \mathrm{Dl})$, recuento de leucocitos mayor que $11{ }^{\star} 10^{9} / \mathrm{L}$ e IMC de $35 \mathrm{~kg} / \mathrm{m}^{2}$ y más. Así, se calculó la puntuación según los siguientes criterios: pacientes de bajo riesgo, resultado $=0$; intermedio, resultado $=1-2$ $\mathrm{u}$ alto riesgo, resultado $\geq 3$ (Tabla 1 ).

\section{Análisis estadístico}

Las variables cuantitativas se reportaron mediante promedios y desviaciones estándar y las cualitativas mediante frecuencias absolutas y porcentajes. También se construyeron gráficos y tablas. El software utilizado para el análisis fue STATA $12^{\circledR}$.

\section{Resultados}

Desde enero a diciembre de 2009, un total de 27 pacientes presentaron un ETE durante la hospitalización u hospitalizados por trombosis en el INC. La edad promedio fue de 59,6 $\pm 1,8$, con $56 \%$ de hombres (Tabla 1). La distribución de las ETE se muestra en la Tabla 2.

Las tres localizaciones más frecuentes fueron: testicular, colon y recto, con $11,1 \%$ respectivamente (Tabla 1).

\section{Tabla 1. Características de los pacientes}

\begin{tabular}{|c|c|}
\hline Edad (años) & 62 años (53-75) \\
\hline \multirow[t]{2}{*}{ Sexo } & $15(55,56 \%)$ \\
\hline & $12(44,44 \%)$ \\
\hline IMC & Mediana: $\quad 24,95(22,14-27,5)$ \\
\hline \multirow[t]{8}{*}{ Diagnóstico oncológico } & Cáncer de colon: \\
\hline & Cáncer de recto: \\
\hline & Cáncer testicular: \\
\hline & Cáncer de mama: 2 (7,41\%) \\
\hline & Cáncer de próstata: 2 (7,41\%) \\
\hline & Cáncer de pulmón: 2 (7,41\%) \\
\hline & Cáncer gástrico: \\
\hline & $10(37,03 \%)$ \\
\hline
\end{tabular}


Las características de los pacientes según la puntuación de Khorama se muestran en las Tablas 3 y 4 .

La distribución de riesgo, según el Modelo de Khorana fue: 4 pacientes clasificaron en bajo riesgo, 20 pacientes en riesgo intermedio y 3 el alto riesgo (Figura1).

Tabla 2. Distribución de ETE

\begin{tabular}{|lcc|}
\hline Tipo ETE & Frecuencia & \% \\
\hline TEP & 1 & 3,70 \\
TVP & 26 & 96,3 \\
\hline Total & 27 & 100 \\
\hline
\end{tabular}

Tabla 3. Parámetros clínicos y de laboratorio de los pacientes según puntuación de Khorama

\begin{tabular}{|lrl|}
\hline Características de los pacientes & n & $(\%)$ \\
\hline Sitio del cáncer & $27(100)$ \\
\hline Muy alto riesgo (estómago, páncreas) & $4(14,8)$ \\
\hline $\begin{array}{l}\text { Alto riesgo (pulmón, linfoma, ginecológico, } \\
\text { vejiga y testicular) }\end{array}$ & $7(26)$ \\
Otros & $16(59,2)$ \\
\hline Plaquetas $>350.000$ & $6(22,2)$ \\
Hb $<10 \mathrm{~g} / \mathrm{dL}$ & $7(26)$ \\
Leucocitos $>11.000$ & $10(37)$ \\
IMC $\geq 35 \mathrm{~kg} / \mathrm{m}^{2}$ & $1(3,7)$ \\
\hline
\end{tabular}

Tabla 4. Modelo predictivo de VTE asociada a la quimioterapia

\begin{tabular}{|lc|}
\hline Características pacientes & Score riesgo \\
\hline $\begin{array}{l}\text { Sitio de cáncer } \\
\text { Muy alto riesgo (estómago-páncreas) }\end{array}$ & 2 \\
$\begin{array}{l}\text { Alto riesgo (pulmón, ginecológicos, } \\
\text { vejiga, testículo) }\end{array}$ & 1 \\
Plaquetas $350 \times 10 \% / \mathrm{L}$ o más & 1 \\
Nivel de $\mathrm{Hb}$ menor a $100 \mathrm{~g} / \mathrm{L}$ & 1 \\
Leucocitos $>11 \times 10^{9} / \mathrm{L}$ & 1 \\
Índice de masa corporal $35 \mathrm{Kg} / \mathrm{m}^{2}$ o más & 1 \\
\hline
\end{tabular}

Fuente: Blood. 2008 May 15;111(10):4902-7. doi: 10.1182/ blood-2007-10-116327. Epub 2008 Jan 23.

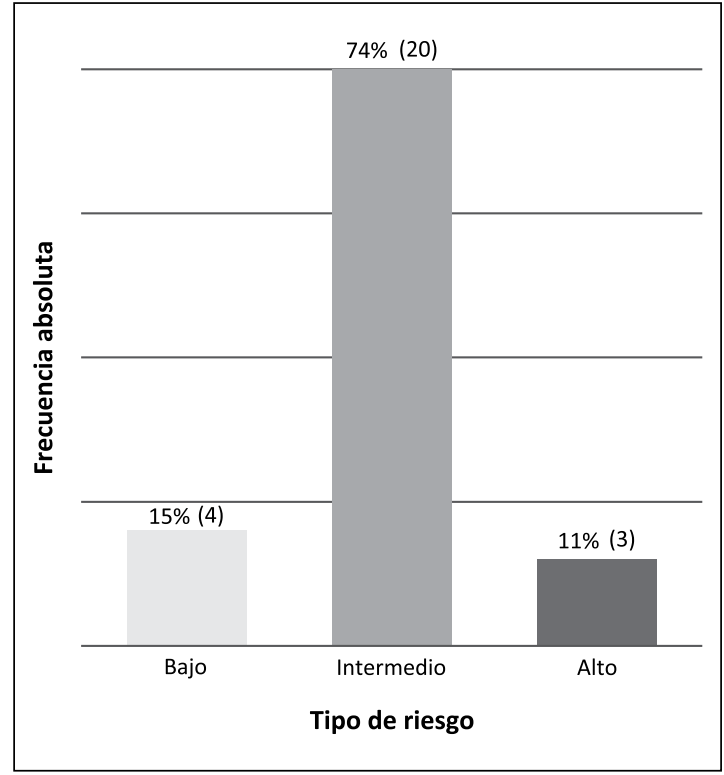

Figura 1. Distribución según modelo de Khorana.

\section{Discusión}

La puntuación de Khorana constituye una escala sencilla que puede ser aplicada al paciente oncológico independiente del tratamiento que esté recibiendo, como fue el caso de este estudio, en donde se presentó un alto porcentaje de pacientes con ETE (80\%), en las categorías de riesgo intermedio y alto. Por lo tanto, la aplicación de esta puntuación con los cinco elementos clínicos a todos los pacientes con cáncer activo, permitiría poner especial énfasis en pacientes con riesgo elevado de desarrollo de ETE y así, enfatizar y promover el correcto uso de las medidas de tromboprofilaxis.

Esta puntuación fue desarrollada para una población de pacientes bajo tratamiento de quimioterapia, pero ha sido validado en diversos estudios en la literatura internacional ${ }^{15-17}$, lo que apoya el valor pronóstico que tendría la aplicación del modelo, no sólo en pacientes con quimioterapia, como fue Mical II diseñado por Khorana. Otro estudio de cohorte retrospectivo, que aplicó la calificación, mostró una mayor incidencia de ETE por cada grupo de riesgo que el estudio original ${ }^{17}$, así como también el estudio CATS ${ }^{16}$. Por ejemplo, el estudio de Ugarte Fornell et al (2013) mostró que el score de Khorana tuvo una sensibilidad y 
exactitud de 20,8\% (IC 95\%: 14,6-28,7) y una proporción de falsos negativos de 79,2\% (IC 95\%: $1,3-85,4)^{18}$. El poder contar con una puntuación predictiva permitirá focalizar o reforzar las medidas de tromboprofilaxis propuestas en las normas internacionales, especialmente en pacientes de mayor riesgo, como son los pacientes oncológicos.

Sin embargo, dada la baja sensibilidad proporcionada por el score, se han agregado dos nuevos biomarcadores dentro de las variables utilizadas en la puntuación de Khorana, siendo estas la P-selectina y dímero $\mathrm{D}^{19-21}$, observándose que los niveles elevados $(\geq 53,1 \mathrm{ng} / \mathrm{ml})$ de la molécula de adhesión celular soluble selectina $\mathrm{P}$ (SP-selectina) se asociaron con un incremento en el riesgo de 2,6 de ETE futuro ${ }^{16}$.

Las mayores limitaciones de este estudio descriptivo fueron el número de pacientes, el cual no cuenta con un tamaño de muestra representativo que permita extrapolar resultados, aunque, el número de casos refleja que el número de pacientes que presentaron un episodio de ETE que se hospitalizaron o diagnosticaron, pero no considera al grupo de pacientes que no consultaron en este centro o fallecieron por esta causa, no siendo diagnosticada.

Otro punto a considerar es la duración del tratamiento y el riesgo de recurrencia de ETE. Respecto a lo último, se han desarrollado algunos modelos para determinar riesgo de recurrencia, pero sólo uno fue desarrollado en pacientes con cáncer ${ }^{22}$. En este aspecto, la puntuación de Khorana podría ser útil para definir la prolongación del tratamiento en aquellos pacientes con riesgo intermedio y alto. En este contexto, el uso del dímero-D como factor adicional en predecir la recurrencia no ha sido aún establecido.

\section{Referencias}

1. Conte G, Figueroa G. Frecuencia de tromboembolismo venoso en pacientes hospitalizados con cáncer. Factores de riesgo y eficacia de la tromboprofilaxis farmacológica. Rev Med Chile 2008; 136: 1528-34.

2. Geerts WH, Pineo GF, Heit JA, Bergqvist D, Lassen MR, Colwell CW, et al. Prevention of venous thromboembolism: the Seventh ACCP Conference on Antithrombotic and Thrombolytic Therapy. Chest 2004; 126 (3 Suppl): 338S-400S.

3. Alikham R, Peters F, Wilmott R, Cohen AT. Fatal pul- monary embolism in hospitalized patients: a necropsy review. J Clin Pathol 2004; 57: 1254-7.

4. Bauer K, Leung L, Tirnauer J. Pathogenesis of the hypercoagulable state associated with malignancy. 2016. Disponible en: http://www.uptodate.com/contents/pathogenesis-of-the-hypercoagulable-state-associated-with-malignancy (Consultado el 10 de mayo del 2016).

5. Heit JA, Silverstein MD, Mohr DN, Petterson TM, O'Fallon WM, Melton L. 3rd. Risk factors for deep vein thrombosis and pulmonary embolism: a population-based case control study. Arch Intern Med 2000; 160: 809-15.

6. Khorana AA, Francis CW, Culakova E, Kuderer NM, Lyman GH. Thromboembolism is a leading cause of death in cancer patients receiving outpatient chemotherapy. J Thromb Haemost 2007; 5: 632-4.

7. Chew HK, Wun T, Harvey D, Zhou H, White RH. Incidence of venous thromboembolism and its effect on survival among patients with common cancers. Arch Intern Med 2006; 166: 458-64.

8. Kröger K, Weiland D, Ose C, Neumann N, Weiss S, Hirsch C, et al. Risk factors for venous thromboembolic events in cancer patients. Ann Oncol 2006; 17: 297-303.

9. Khorana AA, Connolly GC. Assessing risk of venous thromboembolism in the patient with cáncer. J Clin Oncol 2009; 27: 4839-47.

10. Heit JA, Silvestein MD, Mohr DN, Petterson TM, O'Fallon WM, Melton LJ. III Risk factors for Deep vein thrombosis and pulmonary embolism: a population -bases case- control study. Arch Intern Med 2000; 160: 809-15.

11. Collins R, Scrimgeour A, Yusuf S, Peto R. Reduction of mortality in general medical in patients by low-dose heparin prophylaxis. Ann Intern Med 1982; 96: 561-5.

12. Lyman G, Khorana A, Falanga A, Clarke-Pearson D, Flowers C, Jahanzeb M, et al. American Society of Clinical Oncology Guideline: Recommendations for Venous Thromboembolism Prophylaxis and Treatment in Patients With Cancer. J Clin Oncol 2007; 25: 5490.

13. National Comprehensive Cancer Network (NCCN). Guidelines of Cancer-Associated Venous Thromboembolic Disease. Disponible en: https://www.nccn.org/ store/login/login.aspx?ReturnURL=http://www.nccn. org/professionals/physician_gls/pdf/vte.pdf (Consultado el 15 de enero del 2015).

14. Khorana AA, Kuderer NM, Culakova E, Lyman GH, Francis CW. Development and validation of a predictive model of chemotherapy-associated thrombosis. Blood 2008; 111: 4902-6.

15. Khorana AA, Francis CW, Culakova E, Lyman GH. 
Risk factors for chemotherapy-associated venous thromboembolism in a prospective observational study. Cancer 2005; 104: 2822-9.

16. Ay C, Dunkler D, Marosi C, Chirac AL, Vormittag R, Simanek R, et al. Prediction of venous thromboembolism in cancer patients. Blood 2010; 116: 5377-82.

17. Moore RA, Adel N, Riedel E, Bhutani M, Feldman DR, Tabbara NE, et al. High incidence of thromboembolic events in patients treated with cisplatin-based chemotherapy: a large retrospective analysis. J Clin Oncol 2011; 29: 3466-73.

18. Ugarte FG, Otero CR, Ferrer GM, Morillo GR, Elias HT, Jara PL. Predictive Khorana's model in patients with venous thromboembolic disease and cancer. Med Clin (Barc) 2013; 141 (11): 479-81.
19. Ay C, Vormittag R, Dunkler D, Simanek R, Chiriac $\mathrm{AL}$, Drach J, et al. D-dimer and prothrombin fragment $1+2$ predict venous thromboembolism in patients with cancer: results from the Vienna Cancer and Thrombosis Study. J Clin Oncol 2009; 27: 4124-9.

20. Ay C, Dunkler D, Pirker R, Thaler J, Quehenberger P, Wagner $\mathrm{O}$, et al. High D-dimer levels are associated with poor prognosis in cancer patients. Haematologica 2012; 97: 1158-64

21. Polgar J, Matuskova J, Wagner DD. The P-selectin, tissue factor, coagulation triad. J Thromb Haemost 2005; 3: 1590-6.

22. Kyrle PA. Predicting recurrent venous tromboembolism in cancer: is it possible?.Thromb Research 2014; 133 S2: $17-22$. 